\title{
Ultrafast Intensity Modulation via Raman Gain for All-Optical In-Fiber Processing
}

\author{
Geoff Burdge ${ }^{1}$, Shaif-ul Alam, Anatoly Grudinin, Igor Khrushchev ${ }^{2}$, \\ Michael Durkin, Morten Ibsen, and Ian White ${ }^{2}$ \\ Optoelectronics Research Centre, University of Southampton \\ ${ }^{1}$ Laboratory for Physical Sciences \\ 8050 Greenmead Dr., College Park, Maryland 20740 USA \\ ${ }^{2}$ Department of Electronic and Electrical Engineering \\ University of Bristol
}

\begin{abstract}
We present experimental results on ultra-fast intensity modulation using the Raman effect and demonstrate $10 \mathrm{~Gb} / \mathrm{s}$ selective pulse erasure. The technique being both broadband and polarization insensitive offers ultimate speed in excess of $500 \mathrm{~Gb} / \mathrm{s}$. In addition to pulse erasure, this all-optical modulator can shape pulses as a precise, soft aperture scalpel, and create short, dark pulses.
\end{abstract}

As telecommunication technologies mature there has been a drive towards all-optical transparency without the need of optical-to-electrical conversion. In particular, at nodes within a telecommunications system a data header often has to be erased and rewritten for routing purposes. The current approach is based on detection and demodulation of the incoming data signal and then rewriting the appropriate header data to retransmit the packet or asymmetric transfer mode cell. In future systems striving for all-optical transparency, an alternative approach is to use electro-optical modulators capable of operating at bit-rate frequencies. Commercial Mach-Zehnder modulators, which are currently built in $\mathrm{LiNbO}_{3}$, can operate at $10 \mathrm{~Gb} / \mathrm{s}$ and most likely will reach $40 \mathrm{~Gb} / \mathrm{s}$ when traveling wave electrode structures are used; other electrooptic materials, including GaInAs modulators, may be capable of reaching $100 \mathrm{~Gb} / \mathrm{s}$ [1]. However, this performance may be insufficient for future bitrate transparent time-division multiplexed systems that may require nodes to process data at bit rates in excess of $100 \mathrm{~Gb} / \mathrm{s}$. In this paper we demonstrate an all-optical header eraser at 10 $\mathrm{Gb} / \mathrm{s}$. Although the eraser is demonstrated only at $10 \mathrm{Gbit} / \mathrm{s}$ (limited by the laser pulse used in the experiments), the concept is based on the Raman effect with a response time shorter than 10fs in silica fibers and should therefore be capable of operating at bit rates in excess of $500 \mathrm{~Gb} / \mathrm{s}$, ultimately limited by higher-order nonlinear effects.

The proposed modulator originates from the well-known effect of signal depletion due to Raman gain $[2,3]$. Thus, if data signals, which can be either a cw signal or pulses, and 'eraser' pulses are separated in wavelength by the Stokes shift, and co-propagate within telecommunication fiber, then nonlinear interaction will lead to data signal attenuation that is proportional to the Stokes pulse

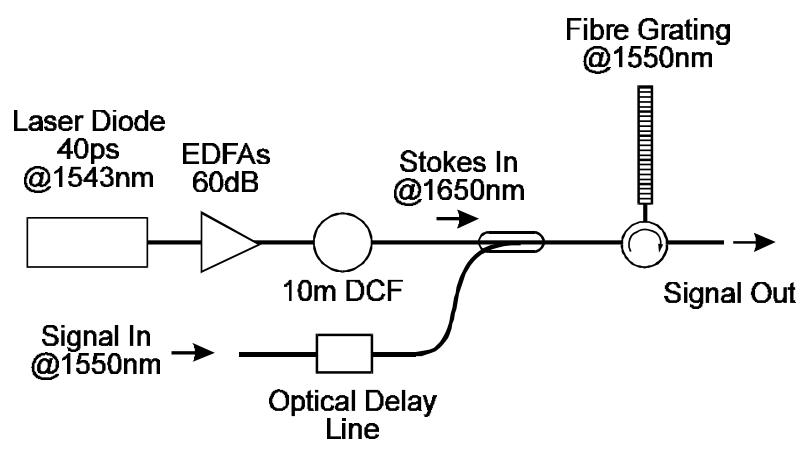

Fig. 1 Block diagram depicting the experimental setup shows generation and detection of the Stokes erasure signal. EDFAs, erbium-doped fiber amplifiers; DCF, dispersion compensating fiber.

intensity and to the interaction length of the two signals within the fiber. Typically, for Stokes power of order of $1 \mathrm{~kW}$ a Raman induced signal loss of $\sim 4 \mathrm{~dB} / \mathrm{m}$ can be achieved. Because the data-signal group velocity is greater, the data signal travels through the Stokes pulses, transferring energy to them and thus creating a hole with width proportional to the interaction length, group-velocity difference and wavelength separation. The erasure time window is about 1.5 $\mathrm{ps} / \mathrm{m}$ for a fiber dispersion of $17(\mathrm{ps} / \mathrm{nm}) \mathrm{km}$, and data signal and Stokes separation of $\sim 100 \mathrm{~nm}$.[4] Recent advances in optical fiber amplifiers 

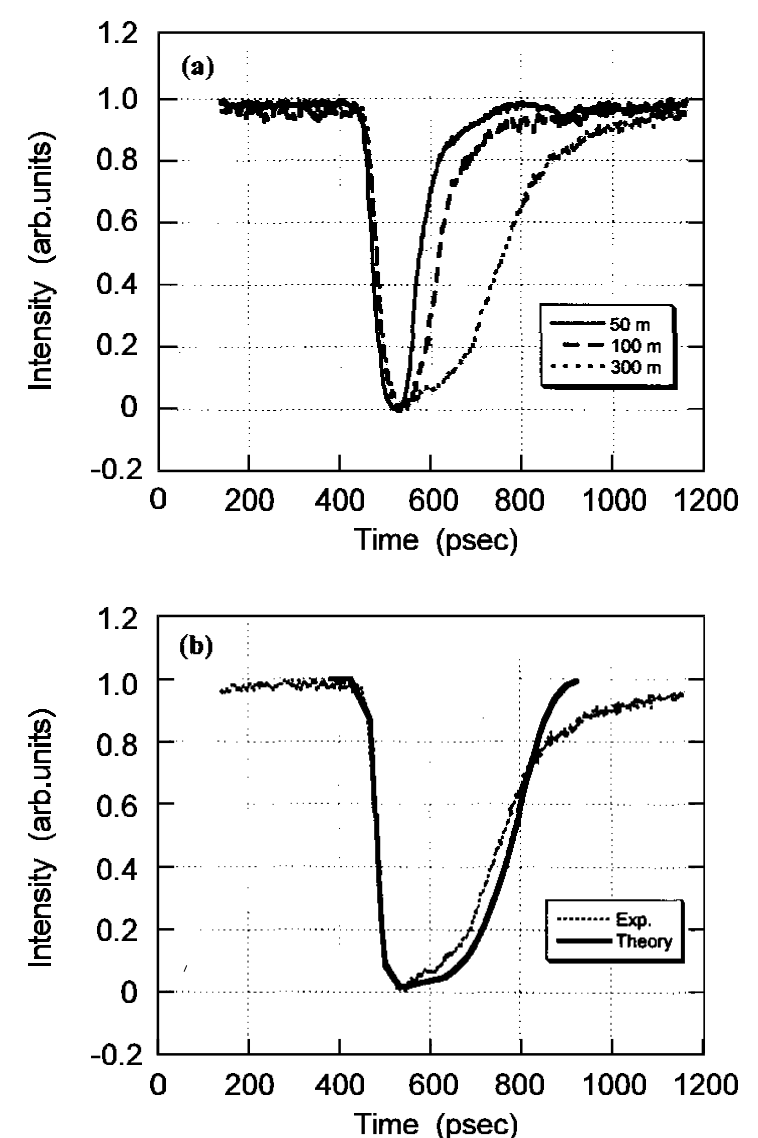

Fig. 2 (a) Examples of hole erasure for 50, 100 , and $300 \mathrm{~m}$ of DSF fiber for Stokesgenerated holes burned in a cw signal at $\mathbf{1 5 4 3}$ nm; (b) a computer simulation incorporating soliton effects is compared to the experimental data for a hole generated in 300m of DSF.

and semiconductor lasers make realization of this technique possible in a rather simple and straightforward way. Fig. 1 depicts the experimental set-up where 40 ps FWHM pulses at $1543 \mathrm{~nm}$ from a gain-switched semiconductor laser diode are amplified by over $60 \mathrm{~dB}$ in a chain of three erbium-doped fiber amplifiers. The high intensity pulses generate red-shifted Stokes pulses at $\sim 1650 \mathrm{~nm}$ by the Raman effect through interaction in a short length $(\sim 10 \mathrm{~m})$ of dispersion compensating fiber (DCF).

Once produced, Stokes erasure pulses are separated from the generating $1543 \mathrm{~nm}$ pulses via a wavelength division multiplexer (WDM). Data pulses are created by either a second synchronized pulsed laser diode operating at $1550 \mathrm{~nm}$ or by residual $1543 \mathrm{~nm}$ pulses. To simulate $10 \mathrm{~Gb} / \mathrm{s}$ data, the laser pulses are passed through a sequence of 50:50 couplers and precise delays to form a four-pulse sequence with pulse separation of less than 100 ps. The four-bit data sequence is then mixed with Stokes erasure pulses via another
WDM and directed into various lengths of dispersion shifted fiber (DSF) with a $\lambda_{0}=1542 \mathrm{~nm}$. The erasure process takes place within the DSF and can be detected after appropriate filtering either by a bulk grating or by a tuned fiber grating and circulator. The use of a $1550 \mathrm{~nm}$ data signal, which is different from the Stokes generating signal, illustrates the broadband nature of the process. The Raman gain bandwidth in silica fiber exceeds $70 \mathrm{~nm}$ around $1550 \mathrm{~nm}$ [5], so the proposed intensity modulator should operate throughout the erbium band from $1535-1565 \mathrm{~nm}$.

The erasure hole width increases monotonically as the DSF fiber interaction length is increased from 25 to $300 \mathrm{~m}$. Fig. 2 illustrates the erasure hole widths achieved for DSF lengths of 50,100, and $300 \mathrm{~m}$ for Stokes-generated holes burned in a cw signal at $1543 \mathrm{~nm}$. One can see the sharp fall time and almost linear increase of the hole width with increasing fiber length. Note that the fall time remains constant, as it is determined by the temporal width of the Stokes pulse, which in this case is 40 ps FWHM. An extinction ratio exceeding $15 \mathrm{~dB}$ was observed, which is sufficient to erase a header before rewriting. Our tests with a polarization controller indicate that the Raman intensity modulator is insensitive to the polarization of either the Stokes erasure pulse or the data pulses, which is due to the fact that the Stokes signal is broadband $(\sim 35 \mathrm{~nm})$, and its polarization is completely scrambled within the short DSF fibers used. ${ }^{6}$

With a group of pulses simulating a 10 Gbit/s data stream we selectively erased by Raman intensity modulation any one data pulse in a fourpulse sequence including two or more consecutive data pulses within the group. Fig. 3(a) demonstrates that any one pulse within a four-bit sequence at $1543 \mathrm{~nm}$ can be erased with excellent extinction ratio without affecting neighboring pulses. We erased each data pulse in the four-bit sequence in $50 \mathrm{~m}$ of DSF by varying the delay between the data sequence and the Stokes erasure pulse. Two consecutive pulses can also be erased as is shown in Fig. 3(b). In this case we formed a longer erasure window by allowing the pulses to interact in $100 \mathrm{~m}$ of DSF. However, as can be seen in Fig. 3, a portion of the fourth data bit was erased along with the desired erasure of bits 2 and 3 of the sequence. This deleterious effect, caused by the slow rising edge of the erasure pulse, is attributed to a multi-soliton break-up effect, and one can circumvent it by exploiting multiple Stokes erasure pulses or greatly alleviate it by using shorter pulses. 

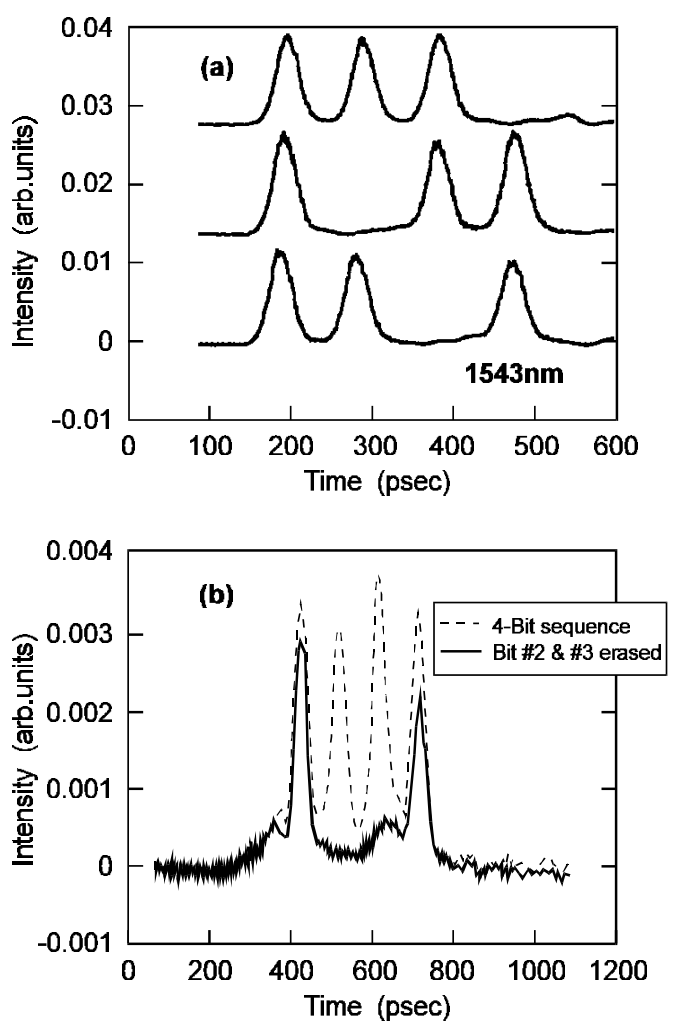

Fig. 3 Data erasure in a four-bit $10 \mathrm{~Gb} / \mathrm{s}$ data sequence, shows (a) selectively erased single bits at $1543 \mathrm{~nm}$ and (b) two consecutive data bits erased at $1550 \mathrm{~nm}$.

Erasure of two consecutive data pulses by generation or multiple Stokes erasure pulses was also experimentally verified ${ }^{4}$. Such a scheme can be extended so that $n$ bits could be erased simultaneously by $n$ equally-spaced Stokes pulses that are matched to the data period.

The fall time and the modulation depth of this intensity modulator are defined by the temporal width of the Stokes pulse and its intensity. In our case the $40 \mathrm{ps}$ Stokes pulse has an intensity as high as $\sim 1 \mathrm{~kW}$, which corresponds to an $N$-soliton pulse with $N \sim 350$ in a fiber with dispersion of $7(\mathrm{ps} / \mathrm{nm}) \mathrm{km}$. Such a high soliton number results in development of modulation instability; that is, the 40-ps pulse tends to split into a number (equal approximately to $N$ ) of fundamental solitons. From our experimental observations we can estimate an individual soliton pulsewidth of $0.5 \mathrm{ps}$, which corresponds to peak power of $\sim 40 \mathrm{~W}$. We can estimate the temporal width of this soliton bunch, which is essentially a noise pulse, to be about 400 ps, which approximately corresponds to the rise time for 300-m fiber shown in Fig.2. Thus, the effect of modulation instability not only broadens the erasure pulse but also reduces its peak power, which in turn leads to lower modulation depth. To verify this effect, we performed numerical
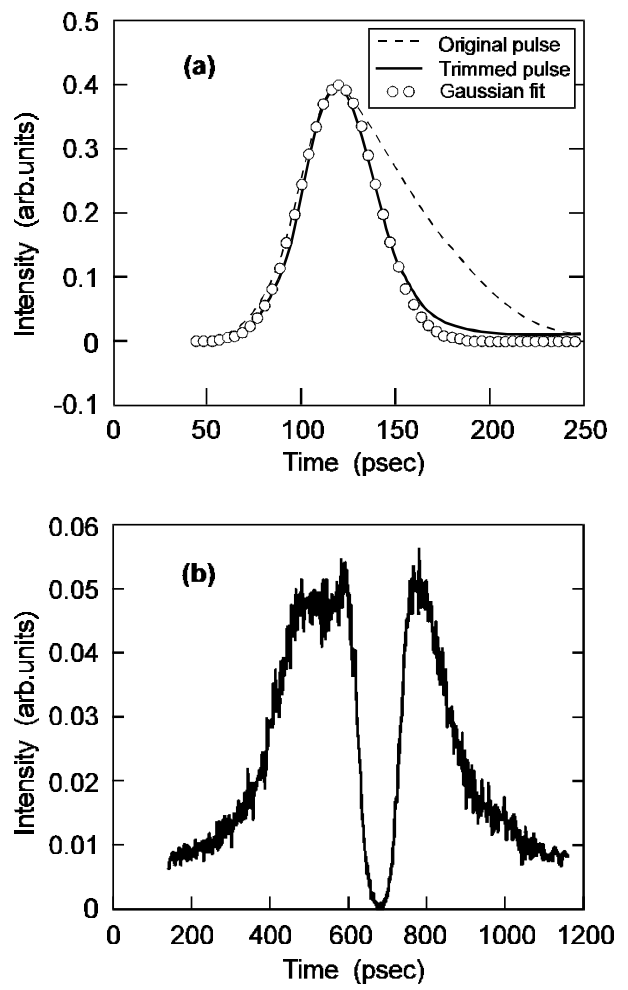

Fig. 4 The Raman modulator as a soft aperture is shown in (a) where an asymmetric pulse has been trimmed. The symmetry of the resulting pulse is illustrated by comparing it to a Gaussian fit. (b) A dark pulse has been formed in an elongated pulse using interaction in $100 \mathrm{~m}$ of DSF.

simulations of nonlinear interactions of Stokes signal pulses, taking into account all the above effects. Fig. 2(b) shows the computed shape of the hole with that obtained experimentally. Reasonably good agreement between the two curves indicates that pulse-width reduction to 1 ps would lead not only to a shorter fall time but also to a shorter rise time, since the number of solitons is greatly reduced. It is also worth mentioning that one can significantly reduce the relatively high intensity and long latency required by the modulator by using new fiber based on gallium-lanthanum sulphide glass, ${ }^{7}$ which possesses $\sim 30$ times higher nonlinear refractive indices and smaller Stokes shifts than that of silica fiber. The smaller Stokes shift has the added benefit that the Stokes pulses can be amplified with standard erbium-doped fiber amplifiers.

This technique also can be used as a timedomain scalpel with which Stokes pulses can be used to precisely trim a signal pulse or to bore a hole in a bright background. The flexibility and usefulness of such a scalpel are shown in Fig. 4(a) which shows the trailing edge of a long, asymmetrical pulse that we modified to create a 
symmetrical pulse by using the Raman intensity modulator as a soft temporal aperture. In Fig. 4(a) the original pulse is contrasted before and after trimming, and the trimmed pulse is compared with a Gaussian fit, illustrating its excellent symmetrical shape.

Another application is depicted in Fig. 4(b) which shows a narrow Stokes pulse that was bored into an elongated pulse. Such a dark pulse can be used to study the propagation dynamics of dark pulses. The use of the technique as a scalpel or as a boring tool indicates that more-complex temporal structures are also possible by controlling the pulse width, fall time and intensity of the Stokes pulses.

We have illustrated ultrafast intensity modulation of optical pulses via the Raman effect in telecommunication fiber. This novel technique is not polarization sensitive, and the broadband Raman gain makes this nonlinear intensity modulator almost wavelength independent. That is, by using the same Stokes pulse at $1650 \mathrm{~nm}$, one can modulate intensity within the entire 1535 $1565 \mathrm{~nm}$ band. The Raman modulator speed, defined by the temporal width of Stokes pulses, should be able to exceed $500 \mathrm{Gbits} / \mathrm{s}$ once the pump pulse is compressed to $\sim 1 \mathrm{ps}$ and most likely will ultimately be limited by higher-order nonlinear effects. Our results show that this technique allows selective erasure of any bit of a pulse sequence including two consecutive bits, trimming of pulses with a soft temporal aperture and boring of a hole into a bright background to produce a dark pulse. Most importantly, this modulation concept allows all-optical modulation of data header signals at rates that can largely exceed projected electrooptical modulation rates.

The research was supported by the US Air Force Office of Scientific Research under grant F617 0896-C0006. We acknowledge helpful comments by D.Mahgerefteh of the Laboratory for Physical Sciences.

\section{References}

1. N. Dagli, University of California, Santa Barbara, 93106, (personal communication).

2. G.P. Agrawal, Nonlinear Fiber Optics, $2^{\text {nd }}$ Edition, (Academic, San Diego, Calif., 1995)

3. P. M. Kjeldsen, M. Øbro, J. S. Madsen, and S. K. Nielsen, Electron. Lett. 32, 1914 (1996).

4. G. Burdge, S.-U. Alam, A. B. Grudinin, M. Durkin, M. Ibsen, I. Khrushchev, and I. White, in Eleventh International Conference on Optics and Optical Fibre Communications and Twenty-Third European Conference on Optical Communication (Institution of Electrical Engineers, London, 1997), paper PD17.

5. R. H. Stolen and E. Ippen, Appl. Phys. Lett. 22, 276 (1973).

6. I. P. Kaminow, IEEE J. Quantum Electron. QE-17, 15 (1981); S. C. Rashleigh, J. Lightwave Technol. 1, 312 (1983).

7. I. Kang, T.D. Krauss, F.W. Wise, B.G. Aitken, and N.F. Borrelli, J. Opt. Soc. Am. B, 12, 2035, (1995). 